\title{
Efficacy of Natural Vinegar and Diluted Lemon Water as a Deparaffinisation Agent in Haematoxylin and Eosin Staining Procedure
}

\author{
Aswani E. ${ }^{1}$, Herald J. Sherlin², Gifirina Jayaraj ${ }^{3}$, Don K.R. ${ }^{4}$, Archana Santhanam ${ }^{5}$ \\ 1,2,3,4,5 Department of Oral Pathology, Saveetha Dental College and Hospitals, \\ Saveetha Institute of Medical and Technical Sciences, Saveetha University, Chennai, Tamilnadu, India.
}

\section{ABSTRACT}

\section{BACKGROUND}

Xylene is the most commonly used deparaffinisation agent as it has excellent clearing properties. However, xylene is not only expensive but also has harmful effects on human health such as hepatitis, chemical pneumonitis, depression, anaemia, etc. Hence, the aim of the study was to compare the efficacy of diluted lemon water and natural vinegar with xylene as deparaffinisation in routine haematoxylin and eosin staining.

\section{METHODS}

Ten histopathologically confirmed cases of pyogenic granuloma blocks were retrieved, sections were made and grouped in to three different groups. Tissue sections in Group I and Group II used diluted lemon water and natural vinegar as deparaffinisation agents respectively and Group III used xylene. Staining characteristics were compared with xylene and scoring was given. A total score of 0 3 was regarded as adequate for diagnosis and less than that as inadequate for diagnosis.

\section{RESULTS}

Adequacy of section adhesion to slide is $100 \%$ for diluted lemon water (DLW) than xylene and natural vinegar and it is statistically significant with a $\mathrm{p}<0.05$. Adequate and comparable nuclear staining and cytoplasm staining of about $90 \%$ DLW and xylene than natural vinegar. All three groups did not show background staining whereas artefacts were considerably more in DLW (50\%) than xylene (40 \%).

\section{CONCLUSIONS}

Eco-friendly deparaffinisation agents such as diluted lemon water demonstrated superior deparaffinisation than routinely used xylene and natural alternatives such as DLW and vinegar can be used as bio-safe alternatives to xylene.

\section{KEY WORDS}

Xylene, Diluted Lemon Water, Natural Vinegar, Deparaffinisation Agent
Corresponding Author: Dr. Herald J. Sherlin, Department of Oral Pathology, Saveetha Dental College and Hospitals, Saveetha Institute of Medical and Technical Sciences, Saveetha University, Chennai-77, Tamilnadu, India.

E-mail: heraldsherlin@gmail.com

DOI: $10.14260 /$ jemds $/ 2020 / 843$

How to Cite This Article:

Aswani E, Sherlin HJ, Jayaraj G, et al. Efficacy of natural vinegar and diluted lemon water as a deparaffinisation agent in haematoxylin and eosin staining procedure. J Evolution Med Dent Sci 2020;9(51):38413845, DOI: 10.14260/jemds/2020/843

Submission 03-09-2020,

Peer Review 28-10-2020,

Acceptance 05-11-2020,

Published 21-12-2020.

Copyright (C) 2020 Aswani E. et al. This is an open access article distributed under Creative Commons Attribution License [Attribution 4.0 International (CC BY 4.0)] 


\section{BACKGROUND}

Histopathology is the gold standard for diagnosis in most surgical cases. Histopathology diagnosis requires tissues to be fixed and processed followed by section cutting and staining. Xylene is the most commonly used deparaffinization agent as it has excellent clearing properties. Commercial xylene is a clear, colourless liquid with aromatic odour. ${ }^{1}$ However, xylene is not only expensive but also has harmful effects on human health such as hepatitis, chemical pneumonitis, depression, anaemia, etc. ${ }^{2}$

Xylene, a mixture of three aromatic hydrocarbon isomers related to benzene, widely used in industries and medical technology as a solvent. It is used in histopathology laboratories for tissue processing, deparaffinization of the tissue sections, cover slipping, cleaning the tissue processors, and recycling. In staining procedures, xylene has excellent dewaxing and clearing capabilities which contribute to brilliantly stain slides. ${ }^{3,4}$

Exposure to xylene can occur via inhalation, ingestion, and eye or skin contact. Long term exposure of xylene may lead to permanent disability caused by diminution of mitochondrial ATP in the affected cells. ${ }^{2-5}$ The National Institute for Occupational Safety and Health recommended exposure limits for xylene at $100 \mathrm{ppm}$ as a time-weighted average for up to a $10 \mathrm{~h}$ work shift and a $40 \mathrm{~h}$ work week and $200 \mathrm{ppm}$ for $10 \mathrm{~min}$ as a short-term limit. Elimination of xylene from tissue processing cuts costs, saves time, and improves the laboratory environment.2,3,6

Many alternatives to xylene have been studied such as alcohols, kerosene, liquid dishwashing solution and mineral oils to overcome the toxigenicity of xylene. Lemon water and natural vinegar are the cheapest, easily available, cost effective, nontoxic and non-inflammable alternative to xylene. Lemon fruit contains a great deal of citric acid which is a weak organic acid. It is a natural preservative and is also used to add an acidic (sour) taste to foods and soft drinks. A solution with a $6 \%$ concentration of citric acid will remove hard water stains from glass without scrubbing. It also serves as an environmentally benign cleaning agent and acts as an antioxidant. $^{7}$

Vinegar contains weak $5-10 \%$ of organic acetic acid is the dominant flavour and an important direct food additive to acidulate food for preservation. Vinegar contains the 4 - $7 \%$ acetic acid and $93-96 \%$ water. In the ratio of $1: 1$, which will produce clearing property which will remove any wax from the glass and even wax from ear during infection. ${ }^{8}$ Xylene can slip down on the ground or even on the surface of water, it will remain for months or more and break down to harmful chemicals. Additionally, it has occupational hazards for humans by soil contamination.9,5 Though various studies have been reported on natural xylene alternatives, in search of newer natural alternatives, no studies have been reported till date including vinegar altering the temperature and time of deparaffinizing agent. Hence, the aim of the study is to compare and evaluate the efficacy of eco-friendly deparaffinization agents such as diluted lemon water and natural vinegar as an alternative to gold standard xylene in histopathological techniques.

\section{METHODS}

\section{Tissue Preparation}

The present study was conducted as a descriptive comparative, pilot study consisting of 10 cases of formalin fixed paraffin-embedded blocks, histopathologically confirmed every consecutive cases of pyogenic granuloma report during the month of June 2019 - June 2020. The blocks were retrieved from the Department of Oral Pathology, Saveetha Dental College. Three sections of $3 \mu \mathrm{m}$ thickness from each block were prepared using a soft tissue microtome (RM 2245) and mounted on albumin coated slides.

The prepared slides were categorised into 3 groups: At 70 degree Celsius in room temperature, $95 \%$ diluted lemon water (Group I), natural vinegar (Group II) and xylene (Group III) were used as deparaffinisation agents and stained with haematoxylin \& eosin. Staining protocol has been mentioned below in Table 1.

\section{Evaluation of Slides}

Two observers who were blinded to the choice of different deparaffinisation agents examined the slides under light microscope (Olympus CH20i) and scoring criteria of the slides were based on the parameters depicted in Table 2.

\section{Statistical Analysis}

All parameters were tabulated in Microsoft Excel sheet and statistical analysis was performed using the SPSS 20.0 version statistical package. As the parameter evaluated are in ordinal scale as Kruskal-Wallis test was done to compare the mean of ranks between the groups and $p<0.05$ was considered to be statistically significant.

\section{RESULTS}

According to the scoring system advocated by Ananthaneni et $\mathrm{al},{ }^{10}$ the average mean score obtained in DLW demonstrated very good adhesion of section to slide with maximum median score of 3.00 and adequacy of $100 \%$ compared to natural vinegar (2.9 with $90 \%$ adequacy) and xylene (2.6 with $60 \%$ adequacy) (Figure 2). DLW and xylene (2.9 with $90 \%$ adequacy) revealed slightly better nuclear staining compared to natural vinegar ( 2.8 with $80 \%$ adequacy). With respect to cytoplasmic staining, xylene had a better score (2.8 with $80 \%$ adequacy) when compared to DLW (2.6 with $60 \%$ adequacy) and natural vinegar ( 2.4 with $50 \%$ adequacy). There was no background staining in all the three groups' studies with a zero-average score. Artefacts were more in the DLW group and had a median score of 2.1 with adequacy of $50 \%$ than xylene and vinegar (1.5 with $40 \%$ adequacy). Overall, there was a significant improvement in adhesion of section to slide using DLW when compared to other groups and was found to be statistically significant. (Kruskal Wallis $p=0.049$ ). The percentage values of the results obtained are represented in Table 3. (Figure 1). 


\begin{tabular}{|c|c|c|c|}
\hline \multirow[b]{2}{*}{ Staining Protocol } & \multicolumn{3}{|c|}{ Time Period for Staining Protocol } \\
\hline & Xylene & $\begin{array}{l}\text { Diluted Lemon } \\
\text { Water (95\%) }\end{array}$ & $\begin{array}{l}\text { Natural } \\
\text { Vinegar }\end{array}$ \\
\hline Incubation period & $30 \mathrm{~min}$ & $30 \mathrm{~min}$ & $30 \mathrm{~min}$ \\
\hline Deparaffinization period & $20 \mathrm{~min}$ & $20 \min .\left(70^{\circ} \mathrm{C}\right)$ & $25 \min .\left(70^{\circ} \mathrm{C}\right)$ \\
\hline Wash with distilled water & - & $5 \mathrm{~min}$. & $5 \mathrm{~min}$. \\
\hline Absolute alcohol & $2 \mathrm{~min}$ & - & - \\
\hline Wash with distilled water & $5 \mathrm{~min}$ & - & - \\
\hline $\begin{array}{c}\text { Lithium bicarbonate } \\
\text { (neutralization of acidity) }\end{array}$ & - & $10 \mathrm{~min}$ & $10 \mathrm{~min}$ \\
\hline Wash with distilled water & - & $5 \mathrm{~min}$ & $5 \mathrm{~min}$ \\
\hline $\begin{array}{l}\text { Staining with Haematoxylin and } \\
\text { Eosin }\end{array}$ & $10 \mathrm{~min}$ & $10 \mathrm{~min}$ & $10 \mathrm{~min}$ \\
\hline Absolute Alcohol & $2 \mathrm{~min}$ & $2 \mathrm{~min}$ & $2 \mathrm{~min}$ \\
\hline Dehydration of slides & $3 \mathrm{~min}$ & $3 \mathrm{~min}$ & $3 \mathrm{~min}$ \\
\hline Mount with DPX and cover slip & same & same & Same \\
\hline $\begin{array}{r}\text { Table 1. Consolidated D } \\
\text { Followed } \\
\end{array}$ & $\begin{array}{l}\text { iction of } \\
\text { the Pre. }\end{array}$ & $\begin{array}{l}\text { fthe Staining P } \\
\text { sent Study }\end{array}$ & edures \\
\hline
\end{tabular}

\begin{tabular}{|cc|}
\hline Parameters & Scoring Criteria \\
1. Adhesion of sections to slides. & 0 - Nil \\
2. Nucleus staining. & 1 - Mild \\
3. Cytoplasmic staining. & 2 - Good \\
& 3 - Very good \\
4. Background staining. & 0 - Absent \\
& 1 - Present \\
5. Artefacts. & 0 - Nil \\
& 1 - Mild \\
& 2 - Moderate \\
& 3 - Severe \\
\hline Table 2. Various Histopathological Parameters and Scoring to Assess \\
the Quality of Sections and Staining (Ananthameni et al ${ }^{10}$ ) \\
\hline
\end{tabular}

\begin{tabular}{|c|c|c|c|c|c|c|}
\hline Parameters & $\begin{array}{c}\text { Types of } \\
\text { Deparaffinization Agent }\end{array}$ & Nil & Mild & Good & $\begin{array}{l}\text { Very } \\
\text { Good }\end{array}$ & $\begin{array}{c}P- \\
\text { Value }\end{array}$ \\
\hline \multirow{3}{*}{$\begin{array}{l}\text { Adhesion of } \\
\text { sections to } \\
\text { slides }\end{array}$} & DLW processing & $0 \%$ & $0 \%$ & $0 \%$ & $100 \%$ & \multirow{4}{*}{$0.049 *$} \\
\hline & natural vinegar & $0 \%$ & $0 \%$ & $10 \%$ & $90 \%$ & \\
\hline & Xylene & $0 \%$ & $0 \%$ & $40 \%$ & $60 \%$ & \\
\hline \multirow{3}{*}{$\begin{array}{l}\text { Nucleus } \\
\text { staining }\end{array}$} & DLW processing & $0 \%$ & $0 \%$ & $10 \%$ & $90 \%$ & \\
\hline & natural vinegar & $0 \%$ & $0 \%$ & $20 \%$ & $80 \%$ & \multirow[b]{2}{*}{0.757} \\
\hline & Xylene & $0 \%$ & $0 \%$ & $10 \%$ & $90 \%$ & \\
\hline \multirow{4}{*}{$\begin{array}{l}\text { Cytoplasmic } \\
\text { staining }\end{array}$} & DLW processing & $0 \%$ & $0 \%$ & $40 \%$ & $60 \%$ & \multirow{4}{*}{0.332} \\
\hline & natural vinegar & $0 \%$ & $10 \%$ & $40 \%$ & $50 \%$ & \\
\hline & Xylene & $0 \%$ & $0 \%$ & $20 \%$ & $80 \%$ & \\
\hline & & \multicolumn{2}{|c|}{ Absence } & \multicolumn{2}{|c|}{ Presence } & \\
\hline \multirow{4}{*}{$\begin{array}{l}\text { Background } \\
\text { Staining }\end{array}$} & DLW processing & \multicolumn{2}{|c|}{$100 \%$} & \multicolumn{2}{|c|}{$0 \%$} & \multirow{3}{*}{1.000} \\
\hline & natural vinegar & \multirow{2}{*}{\multicolumn{2}{|c|}{$100 \%$}} & \multicolumn{2}{|c|}{$0 \%$} & \\
\hline & \multirow[t]{2}{*}{ Xylene } & & & \multicolumn{2}{|c|}{$0 \%$} & \\
\hline & & Nil & Mild & Moderate & Severe & \\
\hline \multirow{3}{*}{ Artifacts } & DLW processing & $10 \%$ & $10 \%$ & $50 \%$ & $30 \%$ & \\
\hline & natural vinegar & $20 \%$ & $30 \%$ & $30 \%$ & $20 \%$ & \\
\hline & Xylene & $10 \%$ & $40 \%$ & $40 \%$ & $10 \%$ & 0.358 \\
\hline \multicolumn{7}{|c|}{$\begin{array}{l}\text { Table 3. Percentage Values of the Parameters Analysed between } \\
\text { Xylene and Natural Alternatives Like Diluted Lemon Water and } \\
\text { Vinegar as a Deparaffinization Agent and with Their Respective P } \\
\text { Ialues (Kruskal Wallis P< } 0.05 \text { Considered as Statistically Significant }\end{array}$} \\
\hline
\end{tabular}

\begin{tabular}{|c|c|c|c|}
\hline & Xylene & $95 \%$ DLW & $\begin{array}{l}\text { Natural } \\
\text { Vinegar }\end{array}$ \\
\hline Cost & High & Low & Low \\
\hline Time taken for staining & $45-50 \mathrm{~min}$ & $55 \mathrm{~min}$ & $60 \mathrm{~min}$ \\
\hline Toxicity & Present & Absent & Absent \\
\hline Biohazardous & yes & no & no \\
\hline Inflammability of chemicals used & Present & Absent & Absent \\
\hline Handling & $\begin{array}{l}\text { Toxic if not } \\
\text { properly handled }\end{array}$ & easy & easy \\
\hline Quality of staining & Good & Good & Good \\
\hline Disposal of chemicals & Difficult & easy & easy \\
\hline Preparation & Synthetic & $\begin{array}{l}\text { Naturally available } \\
\text { from plants }\end{array}$ & $\begin{array}{l}\text { Naturally } \\
\text { available }\end{array}$ \\
\hline \multicolumn{4}{|c|}{$\begin{array}{l}\text { Table 4. Comparison of the Advantages of Xylene, DLW } \\
\text { and Natural Vinegar }\end{array}$} \\
\hline
\end{tabular}
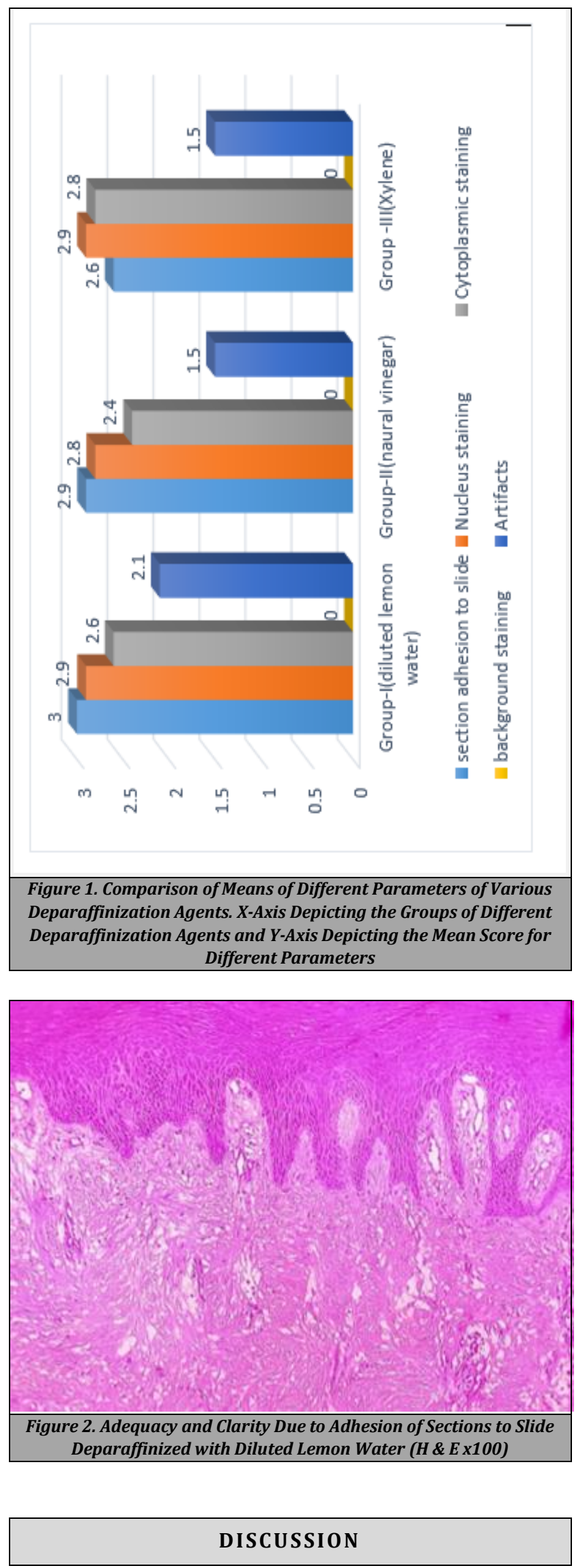

Lemon juice is highly organic with a solvent property containing $5-6 \%$ acetic acid and $\mathrm{pH}$ of 2.2 because of its solvent property it prevents the wax from resticking onto the slides and helping in clearing agents in the sections. ${ }^{10}$ 
Natural vinegar is a clear liquid solution consisting of $5 \%$ acetic acid and 93 - $96 \%$ water. It can be used for cleaning, baking and cooking. It also has health benefits properties such as antioxidant, anti-microbial and anti-tumour activities, etc. ${ }^{8}$ Review of literature showed no study till date where natural vinegar was used as a deparaffinization agent.

Use of xylene in the histology laboratory even after the hazardous effect of xylene became indisputable in the 1970s, many potential substitutes became available. The basis behind the xylene-free method is to produce histological sections equivalent to the conventional ones, but with the advantages of greater economic and environmental gains. ${ }^{11}$

In the present study time taken for the dewaxing using xylene is about $20 \mathrm{~min}$ ( $45 \mathrm{~min}$ for entire staining procedure) which is almost equal to diluted lemon water and for vinegar is $25 \mathrm{~min}$ but overall procedure took more than $50-60 \mathrm{~min}$ which is consistent with result findings of the Kandayala et al in 2014. According to Aparna et al 2018 and Raj et al in 2018 also states the overall time taken for the procedure for xylene was about 50 - $55 \mathrm{~min}$ and DLW about $54 \mathrm{~min}$ which is consistent with our present study. ${ }^{12,1}$ The time period for dewaxing agents of both xylene and DLW shows similar and so DLW can be less time consuming than hazardous xylene.

Nuclear staining is very important in routine staining as it between benign and malignant lesions as identifying features such as nucleoli, hyperchromasia helps to distinguish irregular nuclear membrane and mitotic figures favours a diagnosis of malignancy. ${ }^{4}$ In our study, nucleus staining was better in DLW and xylene group (90\%) when compared to natural vinegar group ( $80 \%$ ), which is similar to the study by Ananthaneni et al. ${ }^{10}(100 \%)$ but it was not similar to the other study by Aparna et al where they reported much inferior results in nuclear staining (75\%). ${ }^{12}$ According to the Wajid Sermadi et al., in their study evaluated the efficacy of coconut oil as a clearing agent about $80 \%$ of nuclear staining and similarly Sudip Indu et al in 2014 stated that only $80 \%$ of nucleus staining capacity. ${ }^{13}, 14$ This shows Diluted lemon water shows better nuclear staining compared to coconut oil and cedarwood oil. Because the coconut oil has the tendency to solidify even in room temperatures and miscibles in nature.

According to Aprana et al in 2018 adhesion of section to the slide for lemon water was $82.5 \%$ and $75.5 \%$ for only coconut and liquid dish wash solution (DLW) which is consistent with the present study show that $100 \%$ of clarity of section adhesion comparable to Xylene and superior to coconut oil and DLW.12 There is also a statistically significant correlation between DLW in the adhesion of the section to slide other than vinegar and xylene $(p<0.05)$. And even natural vinegar $(90 \%)$ has better section adhesion to slides than vegetable oil and xylene. According to Swamy et al, where they tried palm oil, bean oil and vegetable oil show poor quality of staining compared to Xylene which is not consistent with the present study.15,16,17 Although mineral oils and vegetable oil are safe but intensity and uniformity of staining properties is very poor compared to lemon water and natural vinegar. Adequate cytoplasmic staining seen in xylene $80 \%$ than DLW has $60 \%$ and natural vinegar about $50 \%$ this result was similar to Aparna et al studies in that xylene and coconut oil $(100 \%)$ is superior to DLW.12 Even Though the DLW has better quality of adhesion their uniformity among the tissue is less because of some miscible contains of acid in it.

Adequate background staining for all the three groups is the same with $100 \%$, artefacts are more in DLW (50\%) than xylene (40\%). This non-uniformity in staining could be attributed to inadequate deparaffinization, section tears, thick sections and moisture in sections. ${ }^{18}$ In cases of increased time in water bath with DLW the sections would float off whereas reduced time would lead to incomplete dewaxing or it may be because of the organic content of lemon.

Both DLW and natural vinegar have more advantages when compared to xylene like being nontoxic, non-hazardous, easy to dispose of, and less costly. (Table 4) Even though DLW has an added advantage of natural availability, time taken by it to dewax the sections was less (20 min) when compared to natural vinegar (25 min).

During clearing, diluted lemon water with a refractive index of 1.46 - 1.48, closer to that of tissue proteins (varying between 1.33 and 1.4) infiltrates the intercellular spaces of tissues leading to the reduction in the light scattering properties and increase in optical clearance of the tissue making them appear transparent. This indicates that lemon water has easily miscible with water and alcohol which has more clearing properties compared with olive oil and honey as an alternative to xylene. ${ }^{19}$

The disadvantages of Xylene-Free $\mathrm{H}$ and $\mathrm{E}$ staining were that the unique procedure for dewaxing is at 95 degree Celsius temperature sensitive and slight drop in temperature leads to improper removal of wax from sections, and, on the other hand, increase in temperature would lead to lifting up and loss of sections from slides. ${ }^{1}$ It is known that clearing agents has to be miscible with both alcohol and wax but since the diluted lemon water has the properties of maximum displacement of alcohol and makes the tissue become more transparent it could also be used as an alternative clearing agent for xylene in conventional procedure. ${ }^{10}$ However, further studies with large sample sizes and parallel studies focusing on care and optimization of temperature of the deparaffinization agents would yield more concrete results which can be generalised into laboratory practice.

\section{CONCLUSIONS}

Being a pathologist, it is essential to decrease the price and hazards of unsafe chemical agents used in histopathological laboratories. The quality of eco-friendly deparaffinisation agents is more efficient than xylene in $\mathrm{H} \& \mathrm{E}$ (Haematoxylin and Eosin) staining procedure. Aslo, these are harmless, quicker and cost effective. The current study results show that $95 \%$ diluted lemon water can admittedly be used as bio-safe substitute for xylene as a deparaffinisation agent in $\mathrm{H}$ and $\mathrm{E}$ procedure.

Data sharing statement provided by the authors is available with the full text of this article at jemds.com.

Financial or other competing interests: None.

Disclosure forms provided by the authors are available with the full text of this article at jemds.com.

\section{REFERENCES}

[1] Swaroop RBV, Divya C, Harendra KML. Liquid dish wash solution - can it be an alternative in future for the 
expensive and hazardous xylene in hematoxylin and eosin staining of paraffin sections. Tropical Journal of Pathology and Microbiology 2018;4(2):139-43.

[2] Kandyala R, Raghavendra SPC, Rajasekharan ST. Xylene: an overview of its health hazards and preventive measures. J Oral Maxillofacial Pathology 2010;14(1):1-5.

[3] Rai R, Yadav R, Bhardwaj A. Biosafe substitutes to'Xylene': a review. Int J of Information, Research \& Review 2016;3(6):2529-32.

[4] Ghosh S, Rao RS, Nambiar S, et al. Quest for biofriendly Xylene substitutes in histopathology: a comparative study. Journal of International Oral Health 2016;8(12):1101-4.

http://www.ispcd.org/userfiles/rishabh/V8I12/V8I12A 9.pdf

[5] Buesa RJ, Peshkov MV. Histology without Xylene. Ann Diagn Pathol 2009;13(4):246-56.

[6] Buesa RJ, Peshkov MV. Complete elimination of Xylene in practice of a histology laboratory. Arkh Patol 2011;73(1):54-60.

[7] Simonne AH, Ritenour MA. Citrus (Orange, Lemon, Mandarin, Grapefruit, Lime and Other citrus fruits). In: Terry LA, edr. Health-promoting properties of fruits and vegetables. Oxfordshire, UK: CAB International 2011: p. 90-117.

[8] Budak NH, Aykin E, Seydim AC, et al. Functional properties of vinegar. J Food Sci 2014;79(5):R757-R64.

[9] Chen CY, He T, Mao XL, et al. A novel xylene substitute for histotechnology and histochemistry. Biotech Histochem 2010;85(4):231-40.

[10] Ananthaneni A, Namala S, Guduru VS, et al. Efficacy of $1.5 \%$ dish washing solution and $95 \%$ lemon water in substituting perilous xylene as a deparaffinizing agent for routine $\mathrm{h}$ and e staining procedure: a short study. Scientifica (Cairo) 2014;2014:707310.
[11] Buesa RJ. Mineral oil: the best xylene substitute for tissue processing yet? J Histotechnol 2000;23(2):143-9.

[12] Aparna B, Manjunath AB, Mujib ABR, et al. Comparaing the efficacy of dishwash solution, diluted lemon water, coconut oil and xylene as deparaffizing agents for hematoxylin and eosin staining procedure. International Journal of Anatomy and Research 2018;6(2.1):5176-80. http://dx.doi.org/10.16965/ijar.2018.149

[13] Sermadi W, Prabhu S, Acharya S, et al. Comparing the efficacy of coconut oil and xylene as a clearing agent in the histopathology laboratory. J Oral Maxillofacial Pathology 2014;18(Suppl 1):S49-S53.

[14] Indu S, Ramesh V, Indu PC, et al. Comparative efficacy of cedarwood oil and xylene in haematoxylin and eosin staining procedures: an experimental study. J Nat Sci Biol Med 2014;5(2):284-7.

[15] Udonkang M, Eluwa M, Ekanem TB, et al. Bleached palm oil as substitute for xylene in histology. J Pharm Clin Res 2014; 8:8-17.

[16] Swamy SRG, Nandan SRK, Kulkarni PG, et al. Bio-friendly alternatives for xylene - carrot oil, olive oil, pine oil, rose oil. J Clin Diagn Res 2015;9(11):ZC16-ZC8.

[17] Premalatha BR, Patil S, Rao RS, et al. Mineral oil-a biofriendly substitute for xylene in deparaffinization: a novel method. J Contemp Dent Pract 2013;14(2):281-6.

[18] Hamill JR, Spencer S. Histotechnique and staining troubleshooting. In: Morgan MB, Spencer JM, Hamill Jr, et al. eds. Atlas of Mohs and frozen section cutaneous pathology. Cham: Springer International Publishing 2018: p. 213-30.

[19] Rylander CG, Stumpp OF, Milner TE, et al. Dehydration mechanism of optical clearing in tissue. J Biomed Opt 2006;11(4):041117. 